\title{
Publisher Correction: Targeting co-stimulatory molecules in autoimmune disease
}

Natalie M. Edner(D, Gianluca Carlesso (1), James S. Rush and Lucy S. K. Walker(i)

Nature Reviews Drug Discovery https://doi.org/10.1038/s41573-020-0081-9 Published online 16 September 2020

The term CTLA4-immunoglobulin was incorrectly used instead of CTLA4-lg throughout the article. This has been corrected in the online and print versions.

https://doi.org/10.1038/s41573-020-00116-x I Published online 18 November 2020

(C) Springer Nature Limited 2020 